\title{
Pregnancy-associated microangiopathy: From the diagnostic to the treatment of atypical hemolytic-uremic syndrome (aHUS)
}

\author{
Evrard $Z^{1}$, Christophe $\mathrm{JL}^{2}$, Cambier JF ${ }^{2}$, De Selliers de Moranville $\mathrm{I}^{1}$, Cheron $\mathrm{AC}^{3}$ and Mhallem Gziri $\mathrm{M}^{1 *}$ \\ ${ }^{1}$ Department of Gynecology-obstetric, Cliniques Universitaires Saint-Luc, Brussels, Belgium \\ ${ }^{2}$ Department of Nephrology, Grand Hôpital de Charleroi, Charleroi, Belgium \\ ${ }^{3}$ Department of Gynecology-Obstetric, Grand Hôpital de Charleroi, Charleroi, Belgium
}

\begin{abstract}
A 31 years woman underwent an emergency cesarean section at 31 weeks 5 days due to uncontrolled high blood pressure in a pre-eclampsia setting. During the immediate post-partum period, the patient developed thrombotic microangiopathy (TMA) expressedby acute renal failure. The challenge was to diagnose quickly the atypical hemolytic uremic syndrome (aHUS), taking into consideration the severity of renal damage and lack of post-cesarean renal recovery in a pre-eclampsia setting. The aHUS combines several features comprising mechanical hemolytic anemia, thrombocytopenia, and acute renal failure. This clinical condition is accounted for by an either acquired or constitutional dysregulation of the alternative pathway of the complement system. This article reviews the literature concerning pregnancy- and postpartum-related HUS, with particular attention given to differential diagnosis, clinical presentation, promoting factors, associated genetic abnormalities, clinical evolution, as well as treatment of the condition. In a pregnant or post-partum patient afflicted by TMA, It is essential to diagnose HUS. As a matter of fact, initiating a specific treatment with eculizumab, a monoclonal antibody, is a promising treatment that may revolutionize the renal prognosis.
\end{abstract}

What is already known about the topic?

- Pregnancy and post-partum represent up to 35\% of secondary thrombotic microangiopathy (TMA) forms

- Atypical hemolytic-uremic syndrome (aHUS) is a serious medical condition with poor renal prognosis

What does this article bring up for us?

-The publication authors report the differential diagnosis of TMA in the pregnancy setting. In addition, a recent synthesis of HUS classification is proposed

-This article highlights the key elements pertaining to HUS diagnosis, possibly enabling a faster treatment with monoclonal antibodies like eculizumab

\section{Case report}

A 31-year-old non-smoking, primiparous woman, who was at $31+1 / 7$ weeks is hospitalized because of high blood pressure. At admission, the patient reports no headache, scotoma, epigastric bar pain, tinnitus, dizziness, nausea, or vomiting. On clinical examination, no edema of the lower limbs and no abdominal pain are noted. Moreover, the neurological examination is normal, with the exception of a slight bilateral patellar hyperreflexia.

Upon admission, the only biological abnormalities observed are an increase in serum uric acid levels at $8.1 \mathrm{mg} / \mathrm{dL}$ and proteinuria at $400 \mathrm{mg} / \mathrm{dL}$. Other biological parameters are normal: hemoglobin 14 $\mathrm{g} / \mathrm{dL}$, platelets $161,000 / \mathrm{mm}^{3}$, serum creatinine $0.78 \mathrm{mg} / \mathrm{dL}$, and liver transaminase levels within the normal range. Ultrasound reveals a normal fetus estimated at $1500 \mathrm{gm}$, corresponding to the $10^{\text {th }}$ percentile according to the French National College curves.

A treatment with oral alpha-methyldopa, labetalol and magnesium sulfate are initiated. The fetal lung maturation is obtained by betamethasone $12 \mathrm{mg}$, administered twice. During hospitalization, the proteinuria measured over 24 hours was shown to be highly pathological, being estimated at $5584 \mathrm{mg} / 24 \mathrm{~h}$.
The patient remains totally asymptomatic until $31+5 / 7$ weeks LMP, meaning four days after admission. She suddenly reports atypical pain in the right iliac fossa, occipital headaches, visual disturbances and hypertension non-controlled by the treatments. A cesarean section is performed under spinal anesthesia. A 1380 gm girl is extracted; the Apgar score is of 7 at 5 minutes, and 8 at 10 minutes, respectively. The patient does not present any hemorrhage during the delivery. On the first postoperative day, blood pressure is measured at $130 / 50 \mathrm{mmHg}$, and the patient presented with oligoanuria and abnormal biological tests (Table 1).

The patient is, therefore, transferred to intensive care because of her acute renal failure with mechanical hemolytic anemia and moderate thrombocytopenia (TMA) with high suspicion of post-partum HUS.

${ }^{*}$ Correspondence to: Mhallem Gziri M, Department of Gynecology-obstetric, Cliniques Universitaires Saint-Luc, Brussels, Belgium, E-mail: mina.mhallem@ uclouvain.be

Key words: atypical hemolytic-uremic syndrome (aHUS), pregnancy, thrombotic microangiopathy (TMA)

Received: February 15, 2021; Accepted: February 22, 2021; Published: February 25,2021 
Table 1. Biological parameters at postpartum Day 1

\begin{tabular}{|l|c|c|}
\hline Biological markers & Normality ranges & Measured values \\
\hline Potassium $(\mathrm{mmol} / \mathrm{L})$ & $3.5-5$ & 6.35 \\
\hline Serum creatinine $(\mathrm{mg} / \mathrm{dL})$ & $0.6-1.3$ & 3.16 \\
\hline Urea $(\mathrm{mg} / \mathrm{dL})$ & $15-50$ & 73 \\
\hline Platelet count $\left(/ \mathrm{mm}^{3}\right)$ & $150,000-450,000$ & 98,000 \\
\hline Hemoglobin $(\mathrm{g} / \mathrm{dL})$ & $12.2-15$ & 8.6 \\
\hline Lactate dehydrogenase $(\mathrm{LDH})(\mathrm{IU} / \mathrm{L})$ & $<250$ & 1971 \\
\hline Haptoglobin $(\mathrm{mg} / \mathrm{dL})$ & - & $<8$ \\
\hline Aspartate aminotransferase $(\mathrm{AST})(\mathrm{U} / \mathrm{L})$ & $13-35$ & 145 \\
\hline Schizocytes $(/ 1000$ hematics) & - & 13 (or $1.3 \%)$ \\
\hline
\end{tabular}

The laboratory findings, autoimmune serologies are negative, including the search for anti-nuclear factor (ANF), antibodies to neutrophil polynuclear cytoplasm antibodies (ANCA), antiphospholipid antibodies like lupus anticoagulant, anti-beta2-GP antibodies, and anti-cardiolipin antibodies. The homocysteine assay is normal, while the direct and indirect Coombs tests ae negative, as was the search for irregular agglutinins. However, signs of activation of the alternating complement pathway were demonstrated, including C3 complement consumption (C3 decreased $82 \mathrm{mg} / \mathrm{dL}$; C4 $17 \mathrm{mg} / \mathrm{dL}$ normal, C3d $1 \mathrm{mg} / \mathrm{dL}[<1.2], \mathrm{C} 3 \mathrm{c} 60 \mathrm{mg} / \mathrm{dL}[<72-156], \mathrm{C} 3 \mathrm{~d} / \mathrm{C} 3 \mathrm{c}$ index $1.7[<1.4]$, Bc factor $9 \mathrm{mg} / \mathrm{dL}[11-22]$, Bb factor $\mathrm{V} 0.32 \mathrm{mg} / \mathrm{dL}[<0.15]$, $\mathrm{FBb} / \mathrm{FB}$ ratio $\mathrm{V} 3.42(<1,07])$. ADAMTS 13 protease activity is at $82 \%$ of normal values. The coprocultures do not reveal any pathogens, even when using polymerase chain reaction (PCR). Anti-lipopolysaccharide antibodies to verotoxigenic Escherichia coli are negative, as well the kidney ultrasound. These additional findings are highly suggestive of an aHUS.

The patient is treated daily by plasma exchanges and hemodialysis in the intensive care unit. No clinical and clinical response are observed after 6 days and a treatment with eculizimab was initiated at $900 \mathrm{mg}$ weekly, following vaccination against encapsulated bacteria (Meningococcus, H. Influenza of group B, and Pneumococcus) and antibiotic prophylaxis. The subsequent evolution is rapidly favorable with a normalization of hemolysis parameters and resumption of diuresis.

Genetic analysis of the complement factors revealed a pathogenic mutation of factor I (c.772 G>A).

A kidney biopsy is performed and exhibits no longer any active signs of thrombotic microangiopathy, but extensive renal fibrosis. For this reason, eculizimab treatment is discontinued.

The patient is weaned from dialysis treatment almost 20 months after the cesarean section. Whereas her renal function remains severely impaired (estimated glomerular filtration rate [eGFR14 mL/min.1.73 $\mathrm{m}^{2}$ ] according to the MDRD formula), there are nowadays no signs of TMA recurrence.

\section{Discussion}

The different TMAs share both clinical (mechanical hemolytic anemia, thrombocytopenia, and systemic dysfunction) and histological (capillary and arteriolar thrombosis with endothelial and vascular wall abnormalities) features [1]. TMAs can be classified as primary including TTP and aHUS. TMAs can be secondary to various pathologies, such as malignant arterial hypertension, infections (whether or not associated with a verotoxinogenic germ), cancers, autoimmune diseases, drugs, transplants, and pregnancy, with the pregnancy scenario accounting for up to $35 \%$ of secondary TMA forms [2].
When a pregnant or postpartum patient suddenly presents mechanical hemolytic anemia and thrombocytopenia, several diagnoses requiring urgent management must be listed, including hemolysis associated with low platelet and elevated liver enzyme levels (HELLP) syndrome, Thrombotic thrombocytopenic purpura (TTP), HUS, and severe forms of postpartum hemorrhage [3]. While renal damage, which is characterized by acute renal failure, proteinuria, hematuria, and high blood pressure, is systematic in HUS forms, it can also be seen in other TMA types. Renal impairment is usually reversible after childbirth in HELLP syndrome cases. The differential diagnosis is based, among other things, on the protease ADAMTS-13 activity, which is collapsed $(\leq 10 \%)$ in TTP cases. (Figure 1$)$.

HUS is the consequence of endothelial cell aggression, which is either induced by the Shiga toxins of Escherichia coli (SHU-STEC) or mediated by acquired or constitutional dysregulation of the alternative pathway of the complement system (also called aHUS) [4]. HUS can be complicated or even precipitated by various conditions, including malignant arterial hypertension, infections (Streptococcus pneumoniae, Influenza A H1N1, human immunodeficiency virus, and others), cancers, autoimmune diseases, drugs, and transplants. Over the last 10 years, the TMA and HUS classification has been repeatedly modified [5]. It appears that particular HUS forms considered as secondary are instead a real aHUS with pathogenic mutations identified in the genes regulating the alternative pathway of the complement system. Moreover, this concept has been confirmed with HUS occurring during pregnancy or post-partum [6]. In this retrospective multicenter series, currently the largest reported in the literature, the data demonstrate that pregnancy- or postpartum-related HUS and non-pregnancyrelated HUS and aHUS share, indeed, the same clinical and genetic characteristics. Most of the HUS patients, the HUS condition occurred during the third pregnancy trimester and in the postpartum period. HUS was observed during the first pregnancy in slightly over half (58\%) of the cases. At the time of diagnosis, the severity of acute renal failure was so high that $71 \%$ of patients required hemodialysis, with a significantly higher percentage in cases with an identified genetic mutation. The renal prognosis was poor, with half (53\%) of the patients remaining in end-stage renal disease (ESRD). This percentage was, again, significantly higher in cases with an identified genetic mutation. Of these 41 ESRD patients, 24 received a kidney transplant, whereas aHUS recurred in 13 patients (54\%). On the genetic level, a variant of the complement genes was identified in 49 of the 87 patients (56\%), involving the factor $\mathrm{H}$ complement in $30 \%$ and factor I complement in $9 \%$ of cases. The mechanisms by which pregnancy precipitates HUS are still poorly understood. From a therapeutic view, plasma exchanges were employed in $78 \%$ of patients, without any real benefits noted since the risk of terminal renal insufficiency (TRI) remained as high as in patients that were not treated by plasma exchanges. In this series, four patients were treated using eculizumab, which is a monoclonal antibody that blocks the $\mathrm{C} 5$ fraction of complement, thus activating the terminal complement pathway and forming the membrane attack complex (C5b-9). Three of the four patients experienced an improvement in renal function and TMA symptom control. During a normal pregnancy, complement is activated in the placenta, whereas this activation is slowed down by two membrane proteins, comprising CD59 and decay accelerating factor (DAF). In postpartum, the protection provided by these proteins likely disappears, which may explain the higher proportion of HUS that occur in the postpartum period.

The therapeutic management of patients presenting with HUS during pregnancy or postpartum remains poorly codified. As previously 


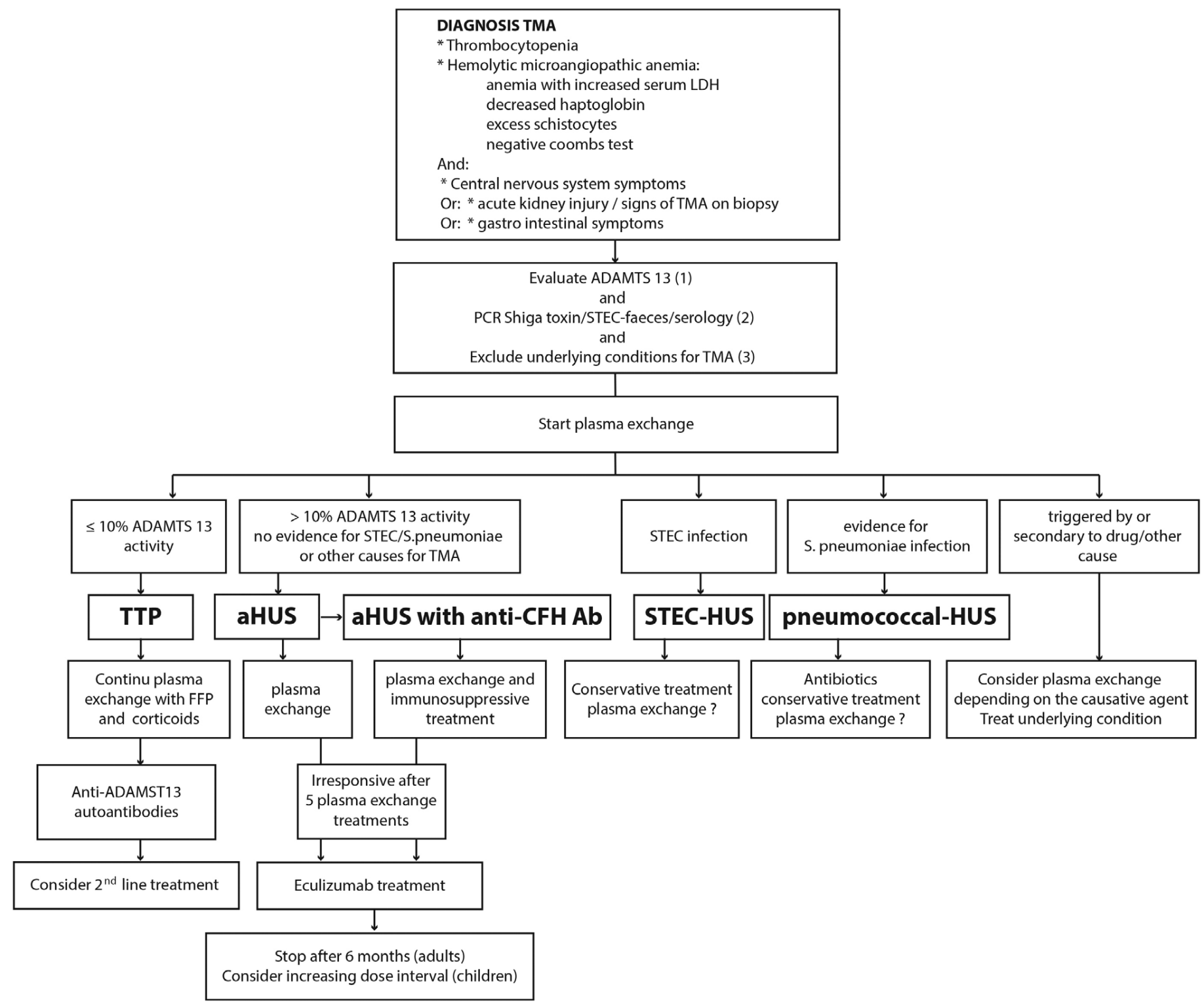

Figure 1. From the diagnostic work-up to the treatment of TMA [10]

explained, plasma exchanges have significant benefits, which has been confirmed in another Spanish retrospective series [7]. The authors of this study evaluated the clinical and prognostic data of 22 pregnancyassociated HUS cases. They revealed the existence of a pathogenic variant of the complement genes in nine of the 22 patients $(41 \%)$. Nearly half of the patients (10/22) were treated using eculizumab, which resulted in a normalization of hematological parameters and preservation of renal function in all cases. It should be noted that three of the 10 patients $(30 \%)$ required emergency hemodialysis prior to eculizumab treatment initiation, and that all in all three patients, renal function actually recovered enabling them to be weaned from dialysis. Conversely, of the 12 patients that were not treated with eculizumab, half developed TRI at the end of follow-up. The authors conclude that the treatment of pregnancy- and postpartum-related HUS should be similar to that of aHUS outside a pregnancy context, meaning that eculizumab should be used in first-line if the clinical presentation, family, or personal medical history is suggestive of HUS or in secondline after plasmapheresis if the diagnosis is uncertain.
Eculizumab is additionally initiated for the treatment of paroxysmal nocturnal hemoglobinuria. In this condition, data on eculizumab exposure during pregnancy indicate that there is no increased risk of fetal malformations or fetal/neonatal toxicity [8]. Due to the higher volume of distribution, it must be noted that eculizumab dosing often needs to be increased during pregnancy in order to avoid insufficient complement blockade [9]. Although access to eculizumab has drastically improved the prognosis of HUS and of pregnancy- and postpartum-related HUS, patients should be informed of the risk of recurrence during a subsequent pregnancy, given that this condition is considered severe with a poor renal prognosis. These pregnancies should be followed in Level 3 maternity hospitals, in close collaboration between obstetricians, nephrologists, pediatricians and intensivists.

\section{Conclusion}

A TMA is characterized by the presence of thrombocytopenia, hemolytic anemia, and systemic dysfunction. Differentiation between aHUS, PTT, pre-eclampsia, and HELLP syndrome appears essential. 
The clinical suspicion of HUS is all the stronger in the event of the following situations:

- Pregnancy-related TMA occurs in the postpartum period

- The clinical picture is dominated by kidney damage

- Signs of TMA persist after childbirth, HUS being a chronic disease, whereas HELLP syndrome has a rapid resolution between 24 and 48 hours after childbirth

- Patient displays a personal history of TMA

- The patient exhibits a family history of TMA or aHUS

- The patient presented with obstetric complications of HELLP syndrome or pre-eclampsia in a previous pregnancy.

A consultation with a nephrologist should be sought immediately if HUS is suspected during pregnancy or post-partum. Indeed, initiating eculizumab treatment appears to revolutionize the renal prognosis of these patients, even if further studies are still necessary in order to confirm the safety of its use during pregnancy.

\section{References}

1. Moake JL (2002) Thrombotic microangiopathies. $N$ Engl J Med 347: 589-600. [Crossref]
2. Bayer G, Von Tokarski F, Thoreau B, Bauvois A, Barbet C, et al. (2019) Etiology and outcomes of thrombotic microangiopathies. Clin J Am Soc Nephrol 14: 557-566. [Crossref]

3. Fakhouri F, Vercel C, Fremeaux-Bacchi V (2012) Obstetric nephrology: AKI and thrombotic microangiopathies in pregnancy. Clin J Am Soc Nephrol 7: 2100-2106. [Crossref]

4. Fakhouri F, Zuber J, Frémeaux-Bacchi V, Loirat C (2017) Haemolyticuraemic syndrome. Lancet 390: 681-696. [Crossref]

5. George JN, Nester CM (2014) Syndromes of thrombotic microangiopathy. $N$ Eng $J$ Med 371: 654-666. [Crossref]

6. Bruel A, Kavanagh D, Noris M, Delmas Y, Wong KS, et al. (2017) Hemolytic uremic syndrome in pregnancy and postpartum. Clin J Am Soc Nephrol 12: 1237-1247. [Crossref]

7. Huerta A, Arjona E, Portoles J, Lopez-Sanchez P, Rabasco C, et al. (2018) A retrospective study of pregnancy-associated atypical hemolytic uremic syndrome. Kidney Int 93: 450-459. [Crossref]

8. Kelly RJ, Höchsmann B, Szer J, Kulasekarararaj A, de Guibert S, et al. (2015) Eculizumab in pregnant patients with paroxysmal nocturnal hemoglobinuria. $N$ Eng $J$ Med 373: 1032-1039. [Crossref]

9. Servais A, Devillard N, Frémeaux-Bacchi V, Hummel A, Salomon L, et al. (2016) Atypical haemolyticuraemic syndrome and pregnancy: outcome with ongoing eculizumab. Nephrol Dial Transplant 31: 2122-2130. [Crossref]

10. Claes KJ, Massart A, Collard L, Weekers L, Goffin E, et al. (2018) Belgian consensus statement on the diagnosis and management of patients with atypical hemolytic uremic syndrome. Acta Clin Belg 73: 80-89. [Crossref]

Copyright: (C2021 Evrard Z. This is an open-access article distributed under the terms of the Creative Commons Attribution License, which permits unrestricted use, distribution, and reproduction in any medium, provided the original author and source are credited. 